\title{
Energy-Aware Preferential Attachment Model for Wireless Sensor Networks with Improved Survivability
}

\author{
Rufei Ma ${ }^{1}$, Erwu Liu ${ }^{1}$, Rui Wang ${ }^{1}$, Zhengqing Zhang ${ }^{1}$, \\ Kezhi Li ${ }^{2}$, Chi Liu ${ }^{3}$, Ping Wang ${ }^{1}$ and Tao Zhou ${ }^{4}$ \\ ${ }^{1}$ School of Electronics and Information, Tongji University, Shanghai, China \\ ${ }^{2}$ Medical Research Council, Imperial College, London, UK \\ ${ }^{3}$ School of Software, Beijing Institute of Technology, Beijing, China \\ ${ }^{4}$ Big Data Research Center, University of Electronic Science and Technology of China \\ [e-mail: mrf007@hotmail.com, erwu.liu@ieee.org, ruiwang@tongji.edu.cn, zhang0zq@ gmail.com, \\ kezhi.li@imperial.ac.uk, chiliu@ bit.edu.cn,pwang@mail.tongji.edu.cn, zhutou@ustc.edu] \\ *Corresponding author: Erwu Liu
}

\begin{abstract}
Recent years have witnessed a dramatic increase in topology research of wireless sensor networks (WSNs) where both energy consumption and survivability need careful consideration. To balance energy consumption and ensure survivability against both random failures and deliberate attacks, we resort to complex network theory and propose an energy-aware preferential attachment (EPA) model to generate a robust topology for WSNs. In the proposed model, by taking the transmission range and energy consumption of the sensor nodes into account, we combine the characters of Erdős -Rényi (ER) model and Barabasi-Albert (BA) model in this new model and introduce tunable coefficients for balancing connectivity, energy consumption, and survivability. The correctness of our theoretic analysis is verified by simulation results. We find that the topology of WSNs built by EPA model is asymptotically power-law and can have different characters in connectivity, energy consumption, and survivability by using different coefficients. This model can significantly improve energy efficiency as well as enhance network survivability by changing coefficients according to the requirement of the real environment where WSNs deployed and therefore lead to a crucial improvement of network performance.
\end{abstract}

Keywords: scale-free, WSNs, complex network 


\section{Introduction}

Due to the convenience of deployment, wireless sensor network (WSNs) have been considered as a promising method to realize information transmission in some severe environments. For example, in warfare and military applications, WSNs are widely deployed to achieve hostile monitoring [1]. The severe environments impose extra challenges on the network designs. One key challenge is the limited energy support of the sensor nodes. Energy exhaustion or failure of a number of sensor nodes is able to destroy the whole network [2]. To conquer these problems, the efficient and effective construction and control of WSNs are very important. The main purpose of the construction and control of a topology is to achieve higher connectivity, more efficient energy use and better survivability. This study aims at providing such a topology for WSNs by using complex network theory.

In 1999, Barabsi and Albert [3] made a breakthrough in network science by proposing the first scale-free model, the Barabasi-Albert (BA) model. Compared with the exponential networks (such as pure random networks) [4], the scale-free network has good performance on robustness against random removal or failure of nodes and also has a short average path length [5], [6]. For WSNs, both robustness and connectivity are important. To increase network connectivity, a typical method is to increase the node density, which means the average distance between nodes becomes smaller. According to [7], the optimum hop-count between a source and a sink should decrease in such high-connectivity networks to achieve maximum energy efficiency. In other words, a scale-free topology is helpful to a WSN's performance, in terms of both robustness and energy efficiency. Because of such benefits, the BA model has recently attracted interest of researchers in WSNs [3],[8]-[12]. Many studies have proposed improved models to balance the energy consumption in WSNs. For example, Zhu et al. [13] proposed an energy-aware evolution model and suggested limiting the number of nodes in every new comer's local-area. However, the local-area in this study is completely irrelevant to the location of nodes, which is practically unreasonable in WSNs. In [14], Zheng et al. proposed the Linear Growth Evolution Model and Accelerated Growth Evolution Model. In these two models, if a cluster head loses a link, it needs to add $n$ new links to other cluster heads for compensation to ensure the connectivity of the network. While this reconstruction mechanism improves the robustness performance under deliberate attacks, it in general requires many compensation links which may deplete the node more quickly.

Considering the above issues, this paper proposes an energy-aware preferential attachment (EPA) model to balance connectivity, energy cost, and survivability of WSNs. Specifically, we divide the topology construction into two phases. In the first phase, we randomly place nodes in a fixed region, and each node is able to get other nodes' information in its transmission range. In the second phase, the network evolves according to preferential attachment and stops until all nodes are added into the network.

Interestingly, we find that setting the coefficients of EPA to some specific values will accordingly reduce it to the BA model, energy-balanced evolution (EAEM) model which only considers energy and degree of node similar to [13], random network model or so-called energy-balanced network (EN) model where energy is the only concern in topology generation. We apply the mean-field method to analyze the EPA model and obtain the degree distribution in a closed form. Both theoretical analysis and simulation have proved that EPA is asymptotically power-law, with a degree exponent different from that of the BA model.

The rest of the paper is organized as follows. In section II, we present the EPA model for 
network topology generation. In section III, we use the mean-field method to derive the degree distribution of EPA. In section IV, we provide simulation results to evaluate the performance of EPA. Section V concludes this paper.

\section{Scale-Free Models: BA and EPA}

In 1999, Barabasi and Albert proposed the first scale-free model, the BA model, which has a power-law degree distribution. This characteristic has been found in many real networks that exhibit strong robustness against random failures and attacks. With the BA model, a network evolves into a scale-invariant state after $t$ time steps by using the mechanisms of network growth and preferential attachment [15], [16]. The algorithm to construct the BA model is presented as follows:

1) Initialization: initialize the network such that it contains an isolated set of $m_{0}$ nodes.

2) Growth: at every time step, add a new node with $m\left(\leq m_{0}\right)$ edges and link the new node to $m$ different nodes in the network.

3) Preferential attachment: connect a new node to node $i$ with the probability $\prod\left(k_{i}\right)$ which depends on the degree $\left(k_{i}\right)$ of node $i$ in a way of

$$
\prod\left(k_{i}\right)=\frac{k_{i}}{\sum_{n} k_{n}},
$$

where $k_{i}$ means the number of edges of node $i$.

The degree distribution of the BA model is obtained as:

$$
P(k)=2 m^{2} k^{-\gamma},
$$

where the degree exponent $\gamma=3$.

In the preferential attachment step of the above algorithm, the new node prefers to connect to nodes with higher degree when joining the network. In real-life networks, incomers may only connect to a few others in a local area for their limited information [17][18]. We cannot directly apply such preferential attachment to WSNs because of the limitation of the transmission range. To generate a scale-free topology for WSNs, we need to modify the preferential attachment step in the BA model in a local area restricted by the transmission range.

In addition, the lifetime of a WSN depends on the residual energy of nodes, which is also considered in our model. While BA has high robustness against random failure of nodes, it is vulnerable to targeted attacks on hub nodes [19], [20]. Conversely, a random network such as ER model has high robustness against deliberate attack. [21] shows general networks which are neither completely random nor scale-free are desirable in the performance against random failure and deliberate attacks. In this study we combine the characters of ER and BA by proposing an energy-aware preferential attachment model to improve the robustness against both random and deliberate attacks. The algorithm to construct the EPA model is presented as follows:

1) Initialization: initialize the network such that it contains $m_{0}$ nodes randomly linked to each other. 
2) Growth: at every time step, add a new node with $m\left(\leq m_{0}\right)$ edges and link the new node to $m$ different old nodes in the network.

3) Preferential attachment: the new node selects $m$ different old nodes to form the new edges with a probability $\prod\left(k_{i}\right)$ with

$$
\prod\left(k_{i}\right)=\left\{b\left[a \cdot \frac{k_{i}}{\sum_{i \in L} k_{i}}+(1-a) \cdot \frac{1}{n_{L}}\right]+(1-b) \cdot \frac{E_{i}}{\sum_{i \in L} E_{i}}\right\} \cdot P(i \in L A),
$$

where $a$ and $b$ are independently tunable parameters with $1 \geq a \geq 0$ and $1 \geq b \geq 0$, respectively, $k_{i}$ and $E_{i}$ are the degree and the residual energy of node $i, L$ is the set of nodes in the new node's transmission range, $n_{L}$ is the number of nodes in the new node's transmission range, LA donates local-area and $P(i \in L A)$ denotes the probability that the new node is in the transmission range of existing node $i$.

According to Eq. (3), the network can have a quite different structure when with different values of $a$ and $b$. Specifically, EPA reduces to the BA model when $a=b=1$ which means that only the node degree dominates the network topology. It has been shown that, with the BA model the network will evolve with rather high interconnectivity, achieving high robustness to random failure. When $a=0$ and $b=1$, EPA reduces to the random network model which has high robustness to deliberate attack. When $b=0$, EPA reduces to EN model which means that only the residual energy decides the network structure, and nodes in this kind of network will have well balanced energy consumption, resulting in significantly improved lifetime of the whole network. Finally, when $a=1$, EPA reduces to the EAEM model which means that both the degree the residual energy decide the network structure. By adjusting $a$ and $b$, one can obtain proper network structures for different applications.

\section{Asymptotic Analysis}

In the first phase of topology construction, nodes are distributed randomly in region $S$. $t+m_{0}$ is the number of nodes after $t$ time steps. We assume that $\mathrm{t} \gg m_{0}$, the node density is then as approximated as

$$
\rho=\frac{m_{0}+t}{S} \approx \frac{t}{S}
$$

Similar to [11], we assume that the residual energy of node $i$ is

$$
E_{i}=E-k_{i} \cdot e,
$$

where $E$ is the initial energy of each node, and $e$ is the energy cost for a node to establish a link, $k_{i}$ is the degree of node $i$. With Eqs. (4) and (5), the total energy of all nodes at step $t$ in the new node's range can be written as 


$$
\sum_{i \in L} E_{n}=\left[E \cdot\left(t+m_{0}\right)-2 m t \cdot e\right] \cdot s / S,
$$

where $s$ is the transmission area of the new node. In Eq. (6), we assume $s$ is big enough so that we can use the average energy to approach the total energy in each transmission area. With $t \gg m_{0}$, Eq. (6) reduces to

$$
\sum_{i \in L} E_{n}=(E t-2 m t \cdot e) \cdot s / S
$$

Since we place nodes randomly and assume that all the nodes have the same transmission range, the probability that the new node is in the transmission range of existing node $i$ can be written as

$$
P(i \in L A)=s / S \text {. }
$$

In the second phase, the network topology grows according to the preferential attachment rule given by Eq. (2). Substituting Eqs. (7) and (8) into Eq. (3), we obtain the preferential attachment probability given as

$$
\prod\left(k_{i}\right)=\left\{\mathrm{b}\left[\mathrm{a} \cdot \frac{k_{i}}{2 m \cdot s \cdot \rho}+(1-a) \cdot \frac{1}{s \cdot \rho}\right]\right\} \cdot \frac{s}{S}+(1-b) \cdot \frac{E_{i}}{E \cdot t-2 m t \cdot e} .
$$

Substituting Eq. (4) into Eq. (9), we have

$$
\prod\left(k_{i}\right)=\frac{[a \cdot b \cdot(E-2 m e)+(\mathrm{b}-1) \cdot e \cdot 2 m] k_{i}+(1-a) \cdot b \cdot 2 m(E-2 m \cdot e)+2 m E \cdot(1-b)}{2 m(E-2 m e) t} .
$$

Next, we apply the mean-field theory [12] to derive the degree distribution of the EPA model. Specifically, the rate equation for the degree of node $i$ can be denoted by

$$
\frac{\partial k_{i}}{\partial t}=m \prod\left(k_{i}\right)
$$

The initial condition is $k_{i}\left(t_{i}\right)=m$ where $t_{i}$ is the time that we need to add node $i$ into the network. We solve the Eq. (11) and obtain

$$
k_{i}\left(t_{i}\right)=\frac{1}{\alpha}\left[\left(\frac{t}{t_{i}}\right)^{\frac{\alpha}{\chi}} \cdot(\alpha m+\beta)-\beta\right],
$$

where

$\alpha=a b(E-2 m e)+(b-1) \cdot e \cdot 2 m, \beta=(1-a) \cdot b \cdot 2 m \cdot(E-2 m e)+2 m E \cdot(1-b)$, $\chi=2(E-2 m \cdot e)$.

Since $1 \geq a \geq 0$ and $1 \geq b \geq 0$, we have $E-2 m e \geq \alpha \geq-2 m e$ and $2 m E \geq \beta \geq 0$. When 
$a=b=1$, we have $\alpha=E-2 m e, \beta=0$, and the EPA model reduces to the BA model. When $b=0$, we have $\alpha=-2 m e, \beta=2 m e$, and residual energy is the only concern in topology generation. When $a=1$, we have $\alpha=b E-2 m e, \beta=2 m E(1-b)$, and the EPA model reduces to the EAEM model.

Assuming that nodes join the network at equal time intervals, the probability density of $t_{i}$ is given by

$$
P\left(t_{i}\right)=\frac{1}{m_{0}+t}
$$

With Eqs. (12) and (13), we have

$$
\begin{aligned}
& P\left\{k_{i}(t)<k\right\}=P\left\{t_{i}>t\left(\frac{\alpha k+\beta}{\alpha m+\beta}\right)^{-\frac{\chi}{\alpha}}\right\} \\
& =1-P\left\{t_{i}<t\left(\frac{\alpha k+\beta}{\alpha m+\beta}\right)^{-\frac{\chi}{\alpha}}\right\} \\
& =1-\frac{t}{m_{0}+t}\left(\frac{\alpha k+\beta}{\alpha m+\beta}\right)^{-\frac{\chi}{\alpha}}
\end{aligned}
$$

From Eq. (14), we obtain the instantaneous degree distribution given as

$$
\begin{aligned}
& P(k)=\frac{\partial P\left\{k_{i}(t)<k\right\}}{\partial k} \\
& =\frac{t \cdot \chi}{\left(m_{0}+t\right) \cdot(\alpha m+\beta)} \cdot\left(\frac{\alpha k+\beta}{\alpha m+\beta}\right)^{-\left(\frac{\chi}{\alpha}+1\right)}
\end{aligned}
$$

When $t \rightarrow \infty$, Eq. (15) reduces to

$$
\begin{aligned}
& P(k)=\frac{\chi}{(\alpha m+\beta)} \cdot\left(\frac{\alpha k+\beta}{\alpha m+\beta}\right)^{-\left(\frac{\chi}{\alpha}+1\right)} \\
& =\frac{\chi}{(\alpha m+\beta)} \cdot\left(\frac{k+\frac{\beta}{\alpha}}{m+\frac{\beta}{\alpha}}\right)^{-\left(\frac{\chi}{\alpha}+1\right)}
\end{aligned}
$$

This distribution can be approximated as $P(k) \propto\left(k+\frac{\beta}{\alpha}\right)^{-\left(\frac{\chi}{\alpha}+1\right)}$, which also satisfies the Mandelbrot law with $c=\frac{\beta}{\alpha}$ [22]. The degree exponent can be written as 


$$
\gamma=\frac{\chi}{\alpha}+1=\frac{2(E-2 m e)}{a \cdot b \cdot(E-2 m e)+(b-1) \cdot e \cdot 2 m}+1 .
$$

\section{Simulation Results}

In the following, we present some simulations by Matlab to verify the analysis developed in this work. The simulation setup is similar to [14], that is, a network totally has $N=1000$ nodes; the initial network has $m_{0}=5$ isolated nodes and at every time step a new node joins the network; the initial energy of each node is $E=10000$, and for each node the energy cost for building up a link is $e=100$. We randomly place nodes in a square region with a size of $S=100 \times 100 \mathrm{~m}^{2}$. At each time step, a new node joins the network and connects to $m$ different old nodes. We assume that $s$ is big enough so that we can apply the average degree approximation in Eq. (5), i.e.,

$$
\frac{S}{S} \gg \frac{m}{N}
$$

\subsection{Connectivity}

According to (18), transmission range $r$ can be written as $r=\sqrt{\frac{Q m}{N} \cdot S \cdot \frac{1}{\pi}}$, where $\mathrm{Q}$ is a positive value which is large enough to obtain (18). Next we investigate the connectivity and energy of the network for $t$ time steps with different values of $a$ and $b$. With $m=3$ and $a=b=1$ (the BA model), we plot in Fig. 1 the degree distribution with different $Q$ after 30 times simulation with each $Q$. From the figure, we observe that when $Q \geq 15$, the analysis and simulation match quite well.

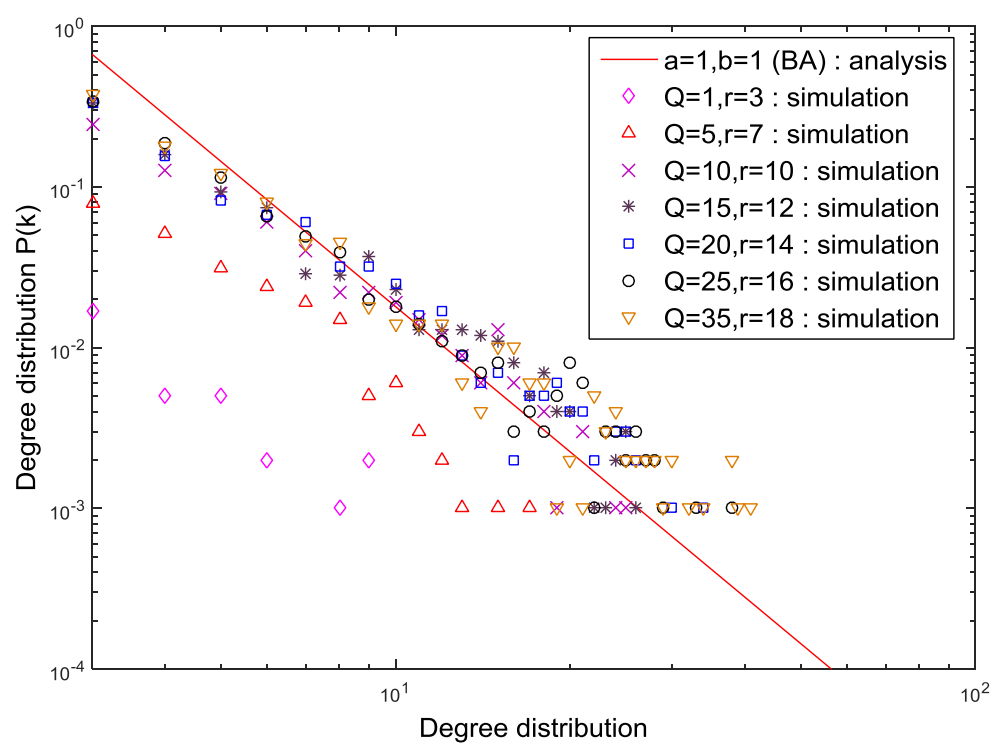

Fig. 1. Degree distribution with different Q 


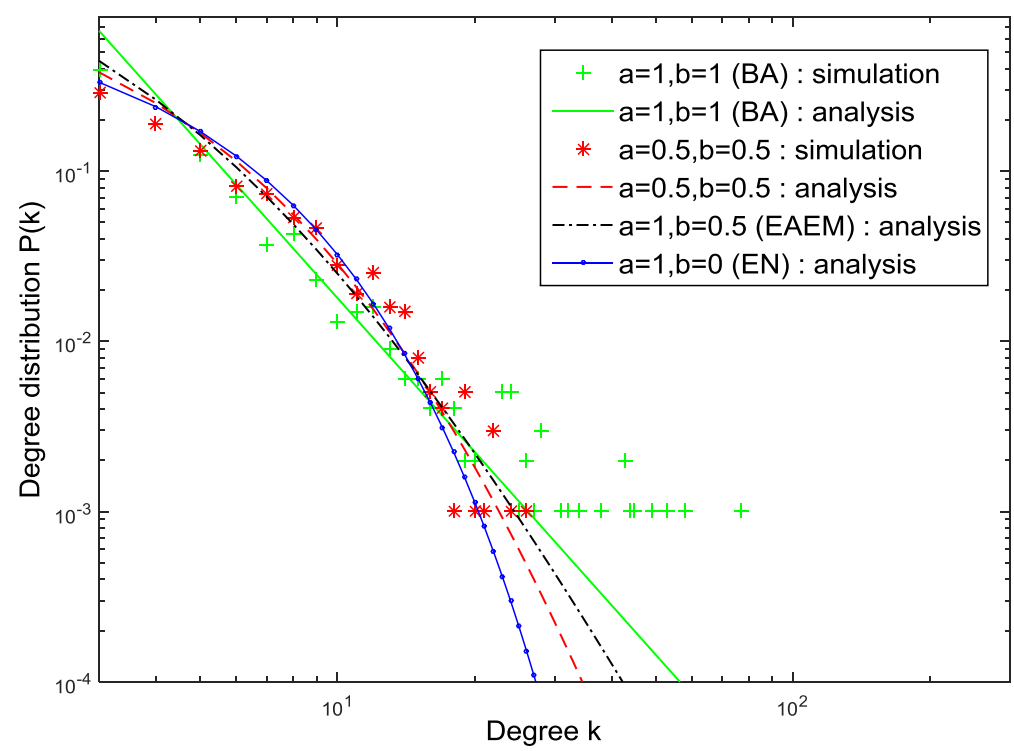

Fig. 2. Degree distribution of EPA with various $a$ and $b$

In the following simulations, we set the transmission range as $r=30 \mathrm{~m}$. Table 1 presents the parameters used in our simulation. With a fixed value of $m=3$, we plot in Fig. 2 the degree distribution with respect to various values of $a$ and $b$. As shown in the figure, both the analysis and simulation verify that EPA is asymptotically power-law. In addition, when we increase the product of $a$ and $b$, the network distribution plot is tightened up from an exponential curve to a power-law line.

Table 1. Parameters for simulation

\begin{tabular}{|c|c|c|}
\hline Parameter & Value & Definition \\
\hline $\mathrm{n}$ & 1000 & Number of nodes \\
\hline $\mathrm{r}$ & $30 \mathrm{~m}$ & Transmission range \\
\hline$m_{0}$ & $100 \times 100 \mathrm{~m}^{2}$ & Entire coverage region \\
\hline $\mathrm{m}$ & 5 & $\begin{array}{c}\text { Number of nodes in the } \\
\text { initial network }\end{array}$ \\
\hline $\mathrm{E}$ & 3 & $\begin{array}{c}\text { Links added in each } \\
\text { time-step }\end{array}$ \\
\hline $\mathrm{e}$ & 10000 & $\begin{array}{c}\text { Initial energy of each } \\
\text { node }\end{array}$ \\
\hline & 100 & $\begin{array}{c}\text { Energy cost for building } \\
\text { up a link }\end{array}$ \\
\hline
\end{tabular}

\subsection{Energy Consumption}

We repeat the simulation for 30 times and depict in Fig. 3 the residual energy for the top-100 most connected nodes with various values of $a$ and $b$. As can be seen, we have more balanced energy consumption of nodes with $a=1$ and $b=0$. On the other hand, when the product $a \cdot b$ increases, we have significantly different energy consumption of the nodes, i.e., 
heavily connected nodes will consume their energy quickly, resulting in highly connected but short-lived nodes. The figure also shows that, if we fix the value of $a \cdot b$, the network will have more balanced energy consumption by decreasing $b$.

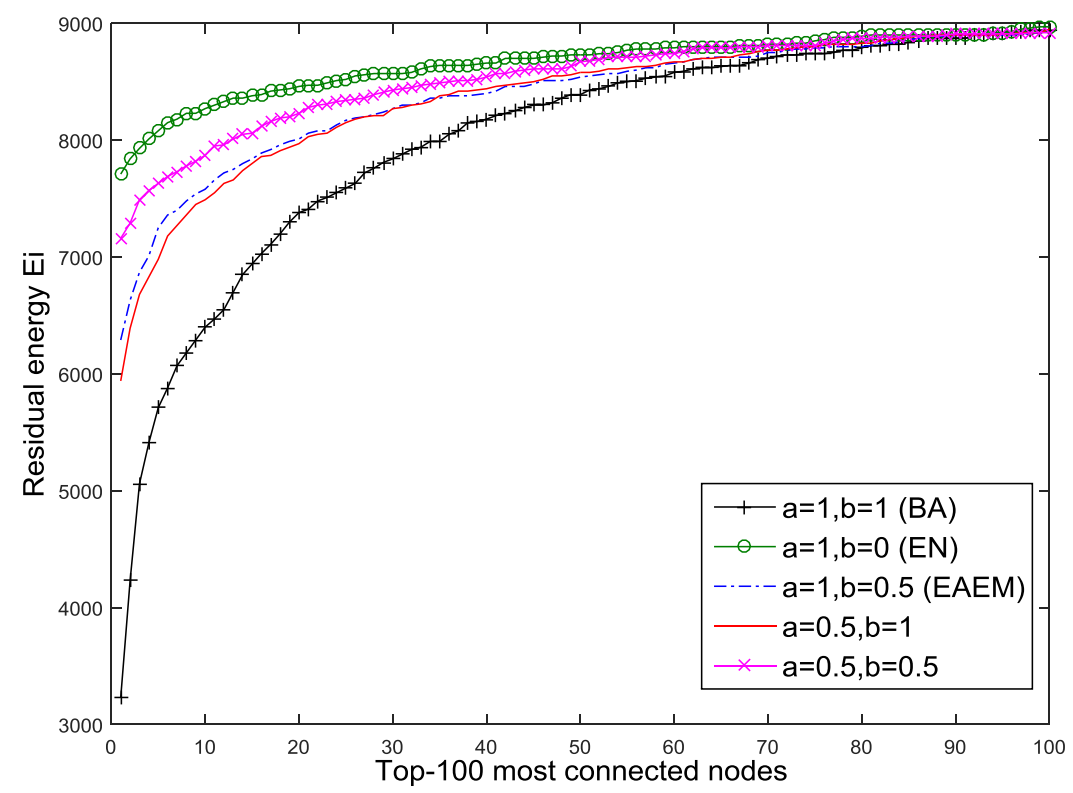

Fig. 3. Residual energy of top-100 most connected nodes

\subsection{Survivability}

Next we use a metric proposed by Zhang et al. [23] to evaluate the survivability of the network under deliberate attacks. Specifically, the metric is defined as

$$
C(T)=\sum_{i=1}^{n} k_{i}(T) /[N(N-1)], \quad T \geq 1,
$$

where $T$ is the maximum number of hops between two nodes, $N$ is network size, $C(T)$ is network coverage, and $k_{i}(T)$ is the number of nodes that one node can reach within $T$ hops. $T$ is set as 7 and we repeat the simulation for 30 times. Fig. 4 plots the simulation results of survivability under deliberate attacks with different settings of $a$ and $b$. As can be seen, the network has better survivability under deliberate attack with a decrease value of $a \cdot b$. When $a \cdot b$ is fixed, the survivability under deliberate attack increases with $b$.

Next we use the network efficiency metric proposed by Crucitti et al. [24] to evaluate the survivability of the network under random failures of nodes, defined as

$$
N E=\frac{1}{N(N-1)} \sum_{i \neq j} \frac{1}{d_{i j}},
$$

where $d_{i j}$ is the number of hops from node $i$ to node $j$. 


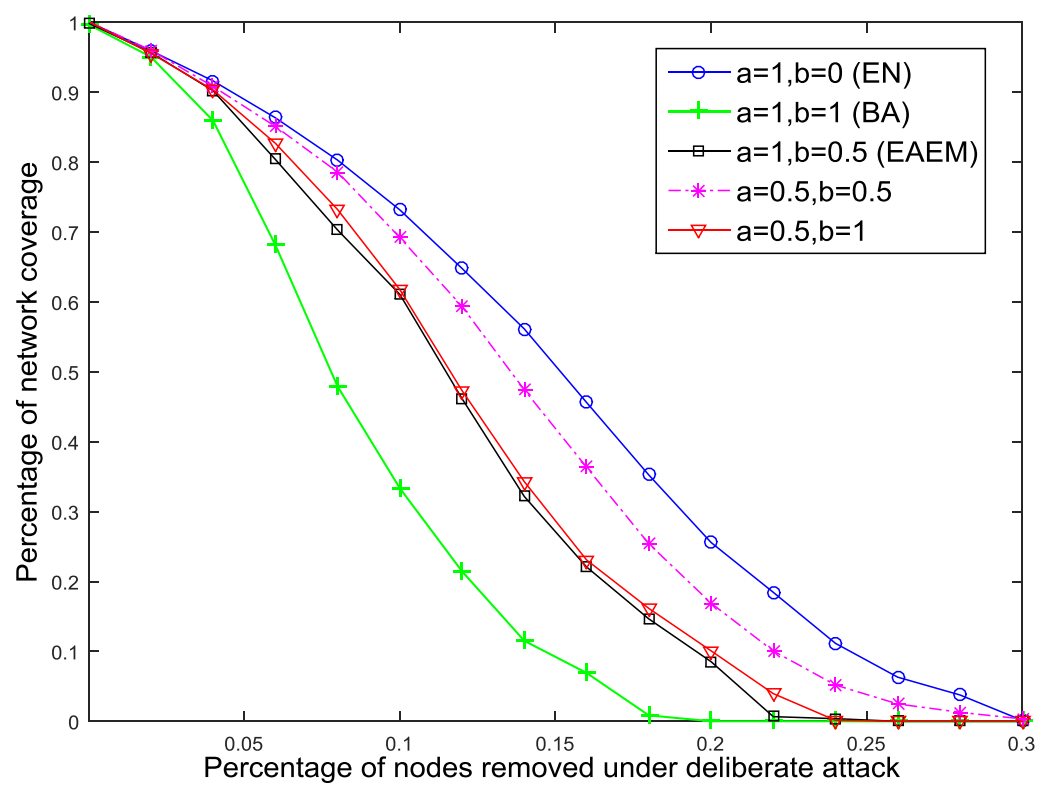

Fig. 4. Survivability of EPA under deliberate attack

We repeat the simulation for 30 times with each deliberate attack percentage and obtain the average values of the network efficiency. Fig. 5 shows the simulation results of survivability under random failure by changing $a$ and $b$. We find that the survivability under random failures increases with the increase of the product $a \cdot b$. The model reduces to BA model when $a=b=1$, which enables it have strong robustness under random attacks. The above analysis shows that, with independent coefficients $a$ and $b$, the proposed EPA model is able to efficiently balance the connectivity, energy consumption and survivability of the WSNs.

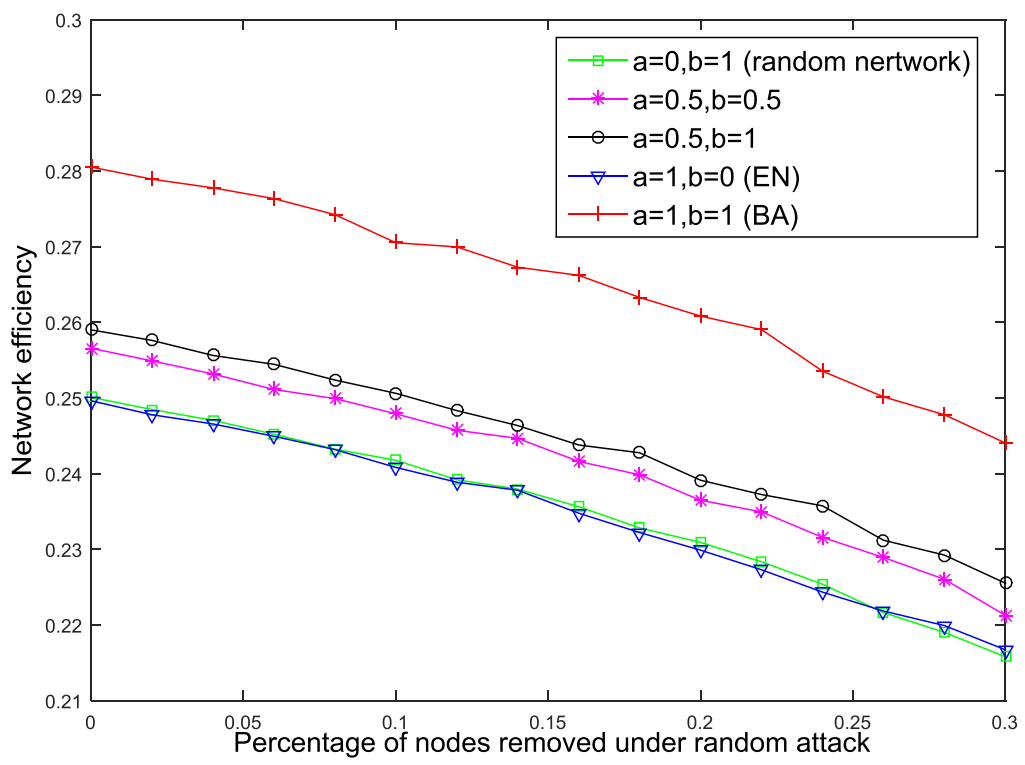

Fig. 5. Survivability of EPA under deliberate attack 


\section{Conclusions}

By using the complex network theory, this paper has proposed for WSNs an energy-aware preferential attachment (EPA) model according to the characteristics of WSNs. The EPA model uses tunable coefficients to adjust the structure of the networks to achieve balance among connectivity, energy consumption, and survivability of WSNs. Theoretical results show that the degree distribution of EPA is asymptotically power-law and simulation results are consistent with the theoretic analysis. In addition, EPA exhibits good performance in robustness against both random and deliberate attacks. In the future, we plan to investigate how to make the best use of the model by exploring the optimal setting of $a$ and $b$.

\section{References}

[1] J. H. Seo, Y. H. Kim, H. B. Ryou, et al, "Optimal sensor deployment for wireless surveillance sensor networks by a hybrid steady-state genetic algorithm," IEICE transactions on communications, vol. 91, no. 11, pp. 3534-3543, 2008. Article (CrossRef Link)

[2] M. Jo, L. Han, N. D. Tan, et al, "A survey: energy exhausting attacks in MAC protocols in WBANs," Telecommunication Systems, vol. 58, no. 2, pp. 153-164, 2015. Article (CrossRef Link)

[3] A.L. Barabási and R. Albert, "Emergence of scaling in random networks," Science, vol. 286, no. 5439, pp. 509-512, 1999. Article (CrossRef Link)

[4] Erdős and Rényi, "On the evolution of random graphs," Selected Papers of Alfréd Rényi, vol. 2, pp. 482-525, 1976.

[5] R. Albert and A.L. Barabási, "Statistical mechanics of complex networks," Reviews of Modern physics, vol. 74, no. 1, pp. 47-74, 2002. Article (CrossRef Link)

[6] A. Fronczak, P. Fronczak, and J. A. Hołyst, "Average path length in random networks," Physical Review E, vol. 70, no. 5, pp. 56-61, 2004. Article (CrossRef Link)

[7] R. Zhang and J.-M. Gorce, "Optimal transmission range for minimum energy consumption in wireless sensor networks," in Proc. of IEEE WCNC, pp. 757-762, 2008.

Article (CrossRef Link)

[8] M. Enachescu, A. Goel, R. Govindan, and R. Motwani, "Scale-free aggregation in sensor networks," Theoretical Computer Science, vol. 344, no. 1, pp. 15-29, 2005. Article (CrossRef Link)

[9] K. Romer and F. Mattern, "The design space of wireless sensor networks," IEEE Wireless Communications, vol. 11, no. 6, pp. 54-61, 2004. Article (CrossRef Link)

[10] D. Wang, E. Liu, Z. Zhang, R. Wang, S. Zhao, X. Huang, and F. Liu, "A flow-weighted scale-free topology for wireless sensor networks," IEEE Communications Letters, vol. 17, no. 2, pp. 235- 238, 2014. Article (CrossRef Link)

[11] Y. Wang, E. Liu, X. Zheng, Z. Zhang, Y. Jian and F. Liu, "Energy-aware complex network model with compensation," in Proc. of IEEE WiMob, Lyon, France, Oct. 2013. Article (CrossRef Link)

[12] A.L. Barabási, R. Albert, and H. Jeong, "Mean-field theory for scale free random networks," Physica A: Statistical Mechanics and its Applications, vol. 272, no. 1, pp. 173-187, 1999. Article (CrossRef Link)

[13] H. Zhu, H. Luo, H. Peng, L. Li, and Q. Luo, "Complex networks-based energy-efficient evolution model for wireless sensor networks," Chaos, Solitons \& Fractals, vol. 41, no. 4, pp. 1828-1835, 2009. Article (CrossRef Link)

[14] G. Zheng, S. Liu, and X. Qi, "Scale-free topology evolution for wireless sensor networks with reconstruction mechanism," Computers \& Electrical Engineering, vol. 38, no. 3, pp. 643-651, 2012. Article (CrossRef Link)

[15] G. Caldarelli, Scale-free networks: complex webs in nature and technology. Oxford University Press, 2007. Article (CrossRef Link) 
[16] R. Albert, H. Jeong, and A.-L. Barabási, “Internet: Diameter of the world-wide web,” Nature, vol. 401, no. 6749, pp. 130-131, 1999. Article (CrossRef Link)

[17] X. Li, and G. Chen, “A local-world evolving network model," Physica A: Statistical Mechanics and its Applications, vol. 328, no. 1, pp. 274-286, 2003. Article (CrossRef Link)

[18] L. Wang, J. Guo, H. Yang and T. Zhou, "Local preferential attachment model for hierarchical networks," Physica A: Statistical Mechanics and its Applications, vol. 388, no. 8, pp. 1713-1720, 2009. Article (CrossRef Link)

[19] A. L. Barabási, R. Albert and H. Jeong, "Error and attack tolerance of complex networks," Nature, vol. 406, no. 6794, pp. 378-382, 2000. Article (CrossRef Link)

[20] L. Li, Q. Jia and H. Wang, "Enhancing the robustness and efficiency of scale-free network with limited link addition," KSII Transactions on Internet and Information Systems (TIIS), vol. 6, no. 5, pp. 1333-1353, 2012. Article (CrossRef Link)

[21] Z. Liu, Y. Lai, N. Ye and P. Dasgupta, "Connectivity distribution and attack tolerance of general networks with both preferential and random attachments," Physics Letters A, vol. 303, no. 5, pp. 337-344, 2002. Article (CrossRef Link)

[22] X. Ren, Z. Yang, B. Wang and T. Zhou, "Mandelbrot law of evolving networks," Chinese Physics Letters, vol. 29, no. 3, p. 038904. Article (CrossRef Link)

[23] M. Zhang, C. Yu, Q. Yang, and T. Quan, "Study on invulnerability of information fusion networks based on network dynamics," Systems Engineering and Electronics, vol. 12, pp. 2421-2424, 2008. Article (CrossRef Link)

[24] P. Crucitti, V. Latora, M. Marchiori, and A. Rapisarda, "Error and attack tolerance of complex networks," Physica A: Statistical Mechanics and its Applications, vol. 340, no. 1, pp. 388-394, 2004. Article (CrossRef Link)

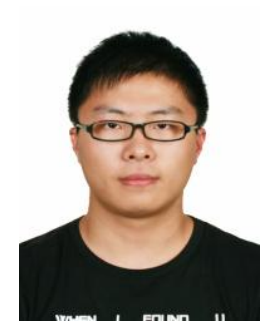

Rufei Ma received his B.E. degree in Communication Engineering from Tongji University, P. R. China, in June 2014. He is currently pursuing a master's degree at the College of Electronics and Information Engineering in Tongji University. His current research interests include complex network theory, wireless sensor networks and heterogeneous networks.

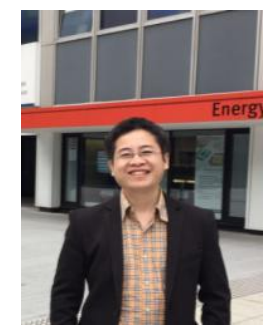

Erwu Liu obtained his Ph.D. in 2001, from the state key laboratory in Huazhong University of Science and Technology. In 2001-2007, he served Alcatel-Lucent as project manager, senior consultant, and senior research scientist. From 2007 to 2011, he was with Imperial College London, working as a research associate. Since 2011, he has been a professor in Tongji University. Professor Liu is an associate editor of the IEEE Communications Letters, and an editor of the KSII Transactions on Internet and Information and Systems. Currently, he is a fellow of the IET, ALTA member of Alcatel-Lucent technical academy, and senior members of both ACM and IEEE. His research interests include complex network theory, wireless sensor networks, deep-penetration communications, high accurate indoor localization, and $5 \mathrm{G}$ technologies. 


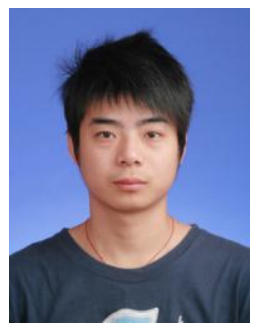

Rui Wang received the B.S. degree from Anhui Normal University, Wuhu, China, in 2006, and the M.S. degree from Shanghai University, Shanghai, China, in 2009 and the Ph.D. degree from Shanghai Jiao Tong University, China, in 2013, all in electronic engineering. From Aug. 2012 to Feb. of 2013, he was a visiting Ph.D student at the Department of Electrical Engineering of University of California, Riverside. From Oct. 2013 to Oct. of 2014, he was with the Institute of Network Coding, The Chinese University of Hong Kong as a postdoctoral research associate. Since Oct. 2014, he has been with the College of Electronics and Information Engineering, Tongji University as an assistant professor. His research interests include wireless cooperative communications, MIMO technique, network coding, and OFDM etc.

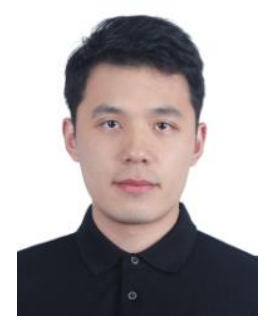

Zhengqing Zhang received his B.E. degree in Automation from Tongji University, Shanghai, P. R. China, in June 2012. He is currently pursuing a doctoral degree at the College of Electronics and Information Engineering in Tongji University. His current research interests include large-scale complex network, wireless magnetic induction communication and heterogeneous networks for complex environment. He currently also serves as a reviewer for a few magazines including IEEE Communications Letters.

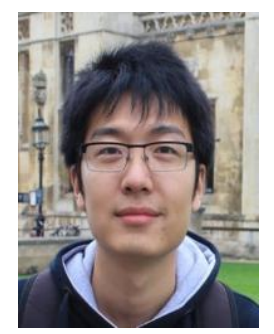

Kezhi (Kenneth) Li is currently a Research Scientist at Medical Research Council (MRC), Imperial College London. He obtained the PhD degree at Imperial College London in 2013, and B.Eng. degree in Electronic Engineering from and University of Science and Technology of China (USTC), Hefei, China, in 2008, respectively. He is a key member in the group of behaviour genomics. His research interests are quite broad in several inter-discipline areas, such as seeking for the relation between organism's genotype and phenotype using computer vision and machine learning, statistical signal processing and their applications in imaging system and quantum tomography, communication and information theory. Before coming to MRC, he used to be a research associate at University of Cambridge, a research fellow at Royal Institute of Technology $(\mathrm{KTH})$ in Stockholm and a research assistant at Microsoft Research Asia (MSRA) and USTC.

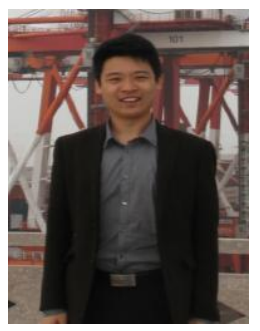

Chi (Harold) Liu receives his Ph.D. degree in Electronic Engineering from Imperial College, London, U.K., in 2010, and his B.Eng. degree in Electronic and Information Engineering from Tsinghua University, Beijing, China, in 2006, respectively. He is currently a Full Professor and serving as the Assistant Dean at the School of Software, Beijing Institute of Technology, China. From 2014, he also serves as the Director of Data Science Institute, the Director of IBM Mainframe Excellence Center (Beijing), the Director of IBM Big Data and Analysis Technology Center, and the Director of National Laboratory of Data Intelligence for China Light Industry. His current research interests include the Internet-of-Things (IoT), big data analytics, mobile computing, and wireless ad hoc, sensor, and mesh networks. 


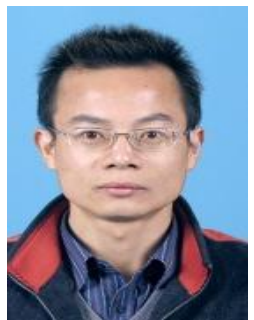

Ping Wang is an associate professor in the department of information and communication engineering at Tongji University. He graduated from the department of computer science and engineering at Shanghai Jiaotong University and received $\mathrm{Ph}$. D. degree in 2007. His main research interests are in routing algorithms and resource allocation of wireless networks (especially for VANETs).

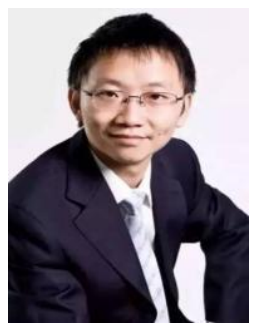

Tao Zhou is a full professor in the University of Electronic Science and Technology of China, and the director of the big data research center. His main research interests include the data mining, network science and collective dynamics. $\mathrm{He}$ has published many research articles in prestigious journals (e.g., Physics Reports, PNAS, Nature Communication), which $>6000$ citations from the Web of Science $(H$-index $=34)$ and $>13000$ citations from the Google Scholar $(H-i n d e x=55)$ until May 2016. 